\title{
7-Level Semi Cross Switched Multilevel Inverter Fed Induction Motor Drive
}

\author{
Srinivas Rao Janiga ${ }^{1, *}$, and Suresh Kumar Tummala ${ }^{2}$ \\ ${ }^{1}$ Assistant Professor, EEE Department, Anurag Engineering College, Kodad, India \\ ${ }^{2}$ Professor, EEE Department, Gokaraju Rangaraju Institute of Engineering \& Technology, Hyderabad, India
}

\begin{abstract}
Multilevel inverters have more prominent features than 2-level inverter due to various advantages like voltage quality, low EMI etc. The semi cross switched multilevel converter topology need less number of semiconductor switches compared to cascaded H-bridge multilevel inverter, and can be implemented to any number of voltage levels. The operating modes of 7-level semi cross switched multi level inverter are discussed. Three phase seven level inverter fed induction motor is implemented in MATLAB/SIMULINK.
\end{abstract}

\section{Introduction}

Now a day's multilevel inverters grab the attention of researchers due to the various advantages like quality output waveform, low EMI, low THD and are suitable for low and medium voltage industrial applications. Many multi-level inverter topologies are proposed and popular among them are the neutral point clamped [2], [3], flying capacitor [4], and cascaded H-bridge [5] structures, neutral point clamped and Flying capacitor multilevel inverters require complex circuitry with the increase in number of levels.

The main topologies of cascade $\mathrm{H}$ bridge is symmetrical with equal voltage sources and asymmetrical with unequal DC sources. The problem with asymmetrical topologies is that some switches have to process through main part of voltage so some high voltage switches are required.

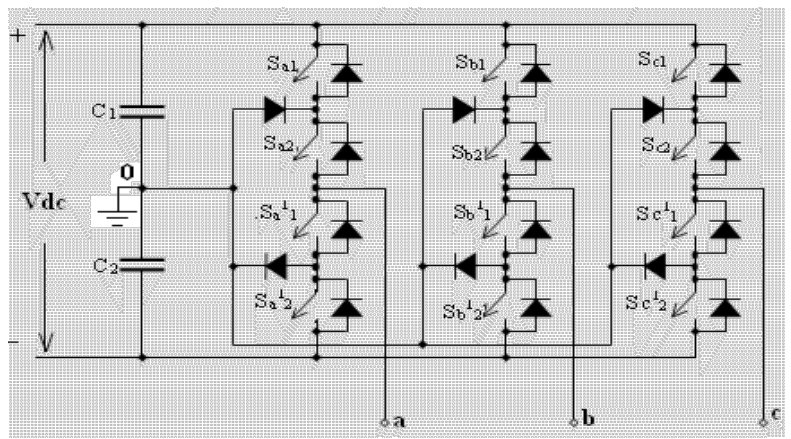

Fig. 1. 3-Phase 3-level Capacitor-Clamped MLI

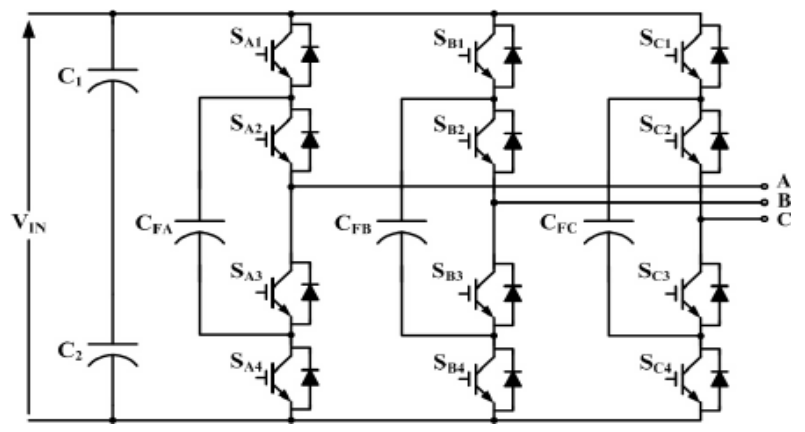

Fig. 2. 3-Phase 3-level Diode-Clamped MLI

\section{Cascaded H-bridge Multilevel Inverter}

The cascaded H-bridge $(\mathrm{CHB})$ inverters are more interested due to the greater demand of medium-voltage high-power inverters. Full bridge strings are connected in cascaded form with separate dc sources to form CHB. Each full- bridge string generates three voltages at the output $+V_{\mathrm{dc}}, 0$ and $-\mathrm{V}_{\mathrm{dc}}$.

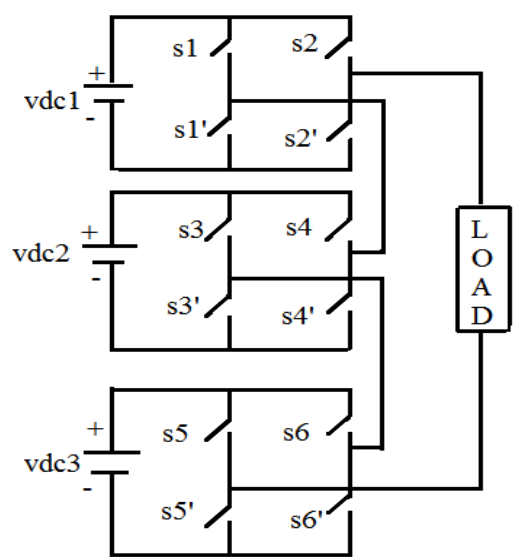

Fig. 3. Single Phase Cascaded H-bridge 7-level Inverter

\footnotetext{
*Corresponding author: janigasrinivasrao@gmail.com
} 


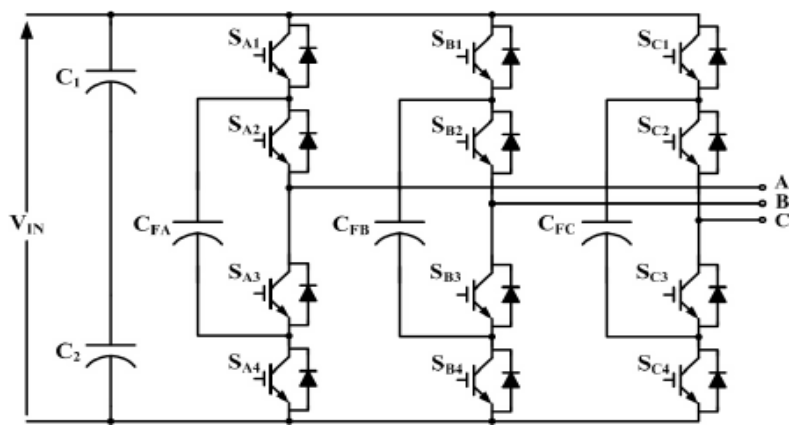

Fig. 4. 3-Phase 3-level Diode-Clamped MLI

Table1: Switching table for 7-level CHB MLI

\begin{tabular}{|c|c|c|}
\hline MODE & Switches ON & $\begin{array}{l}\mathrm{O} / \mathrm{P} \\
\text { Voltage }\end{array}$ \\
\hline 1 & $S_{1}^{\prime} S_{2} S_{3} S_{4} S_{5} S_{6}$ & $V_{d e}$ \\
\hline 2 & $S_{1}^{a} S_{2} S_{3}^{a} S_{4} S_{5} S_{6}$ & $2 V_{d e}$ \\
\hline 3 & $S_{1}^{2} S_{2} S_{3}^{2} S_{4} S_{5}^{2} S_{6}$ & $3 V_{d e}$ \\
\hline 4 & $S_{1} S_{2} S_{3} S_{4} S_{5} S_{6}$ & $0 V_{d e}$ \\
\hline 5 & $S_{1} S_{2}^{i} S_{3}^{u} S_{4}^{u} S_{5}^{u} S_{6}^{i}$ & $-V_{d e}$ \\
\hline 6 & $S_{1} S_{2}^{u} S_{3} S_{4}^{u} S_{5}^{u} S_{6}^{2}$ & $-2 V_{d e}$ \\
\hline 7 & $S_{1} S_{2}^{u} S_{3} S_{4}^{a} S_{5} S_{6}^{a}$ & $-3 V_{d e}$ \\
\hline
\end{tabular}

\section{SEMI CROSS SWITCHED MULTI LEVEL INVERTER}

In this topology the switches are connected as shown in figure5 and this topology requires less number of switches compared to CHB. For 7-level inverter the number of switches required is only seven i.e S1, S2, S3,S1', S2', S3', S4' Therefore, the complexity in driver circuit, number of switches required, number of switches conducting, THD and efficiency [1] reduces. So size and cost of the inverter is reduced compared to $\mathrm{CHB}$ multilevel inverter.

DC Voltage sources V1(100v), V2(100v), V3(100v) for one phase are connected as shown fig. 5. The switches switched as per requirement of voltage level.

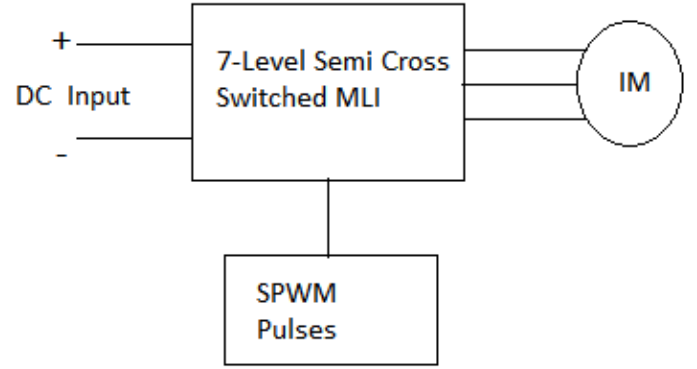

Fig.5. Block Diagram of 7-level semicross switched MLI fed induction motor drive

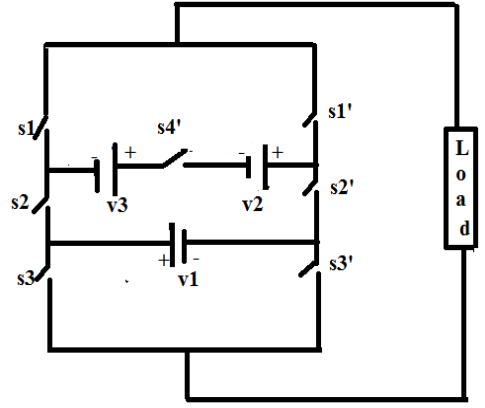

Fig.5. Single phase semi-cross switched multi level inverter

\subsection{Modes of Operation}

The switching operation of 7-level semi cross switched multilevel inverter shown below

Mode-1:

$>$ The switches s1, s2 and s3' are in ON state.

$>$ The voltage across this load is +vdc.

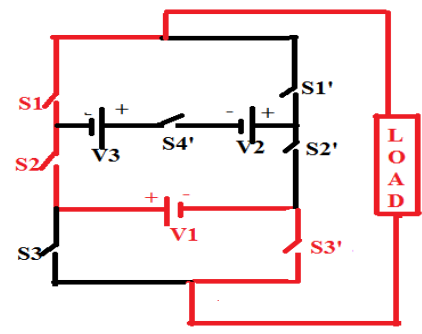

Mode-2:

$>$ The switches s1, s2', s3' and s4' are in ON state.

$>$ The voltage across the load is $+2 \mathrm{vdc}$.

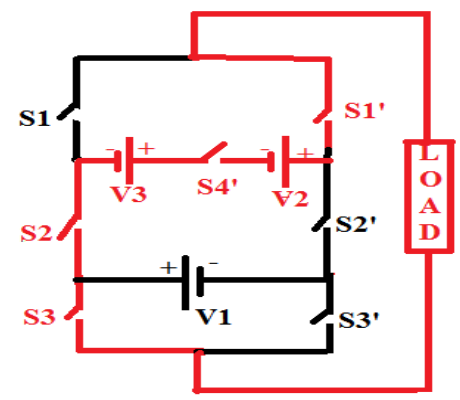

Mode-3:

$>$ The switches $\mathrm{s} 2, \mathrm{~s}^{\prime}, \mathrm{s}^{\prime}$ ' and s4' are in ON state.

$>$ The voltage across the load is $+3 \mathrm{vdc}$.

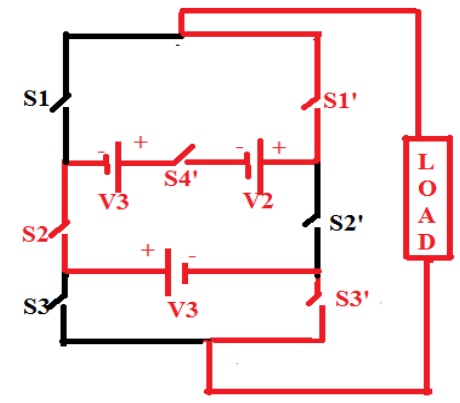


Mode-4:

$>$ The switches s1, s2, and s3 are in ON state.

$>$ The voltage across this load is 0 .

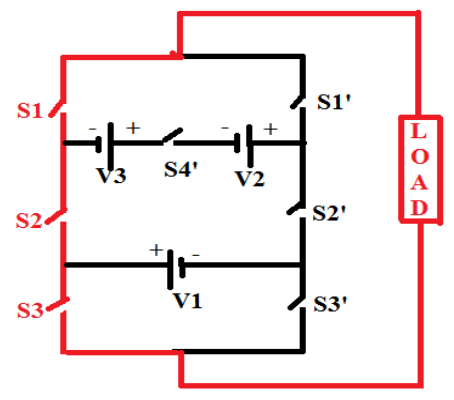

Mode-5:

$>$ The switches $\mathrm{s} 3, \mathrm{~s} 1$ ' and s2' are in ON state.

$>$ The voltage across this load is - vdc.

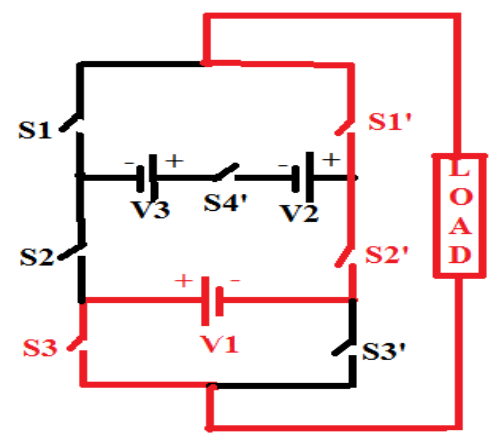

Mode-6:

$>$ The switches s2, s3, s1'and s4'are in ON state.

$>$ The voltage across this load is $-2 \mathrm{vdc}$.

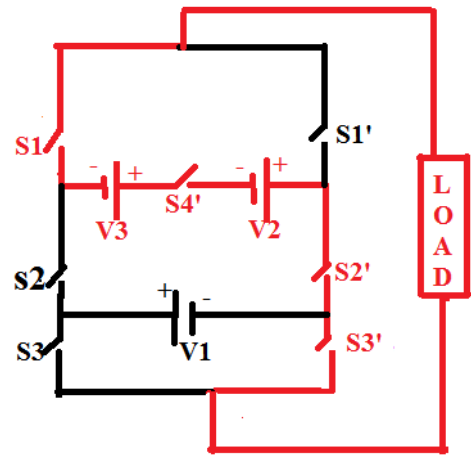

Mode-7:

$>$ The switches s1, s3, s2'and s4' are in ON state.

$>$ The voltage across this load is $-3 \mathrm{Vdc}$.

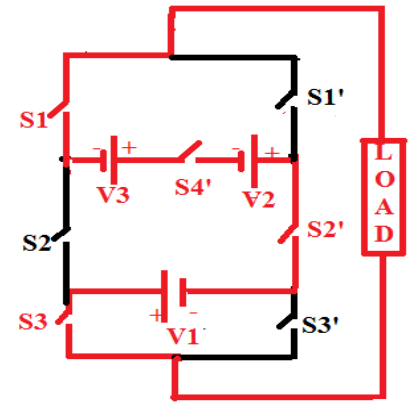

\section{Results}

Simulation of three phase 7-level cascaded $\mathrm{H}$ bridge multilevel inverter and semi cross switched multilevel inverter induction motor is performed using MATLAB/SIMULINK environment.

\subsection{Three phase 7-level cascaded H-bridge multilevel inverter fed IM}

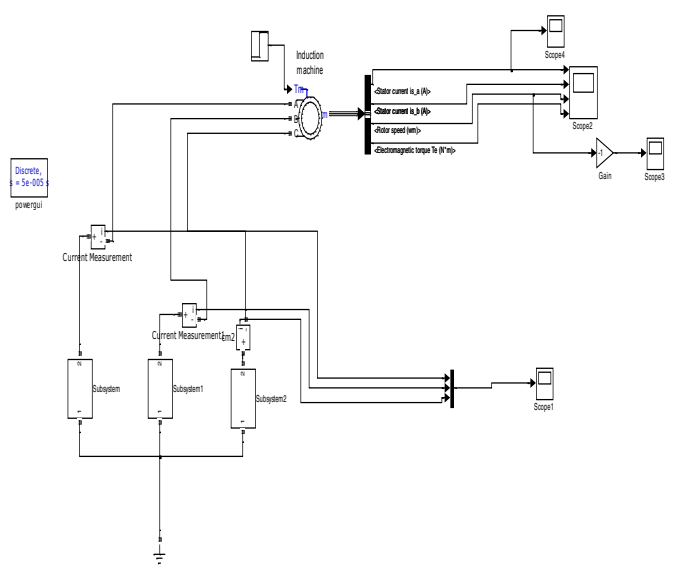

Fig.6. Simulink diagram of Three phase 7-level Cascaded H-bridge multilevel inverter fed IM

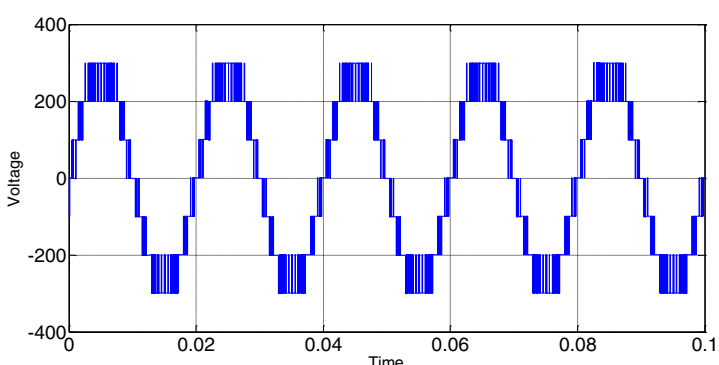

Fig.7. Phase Voltage

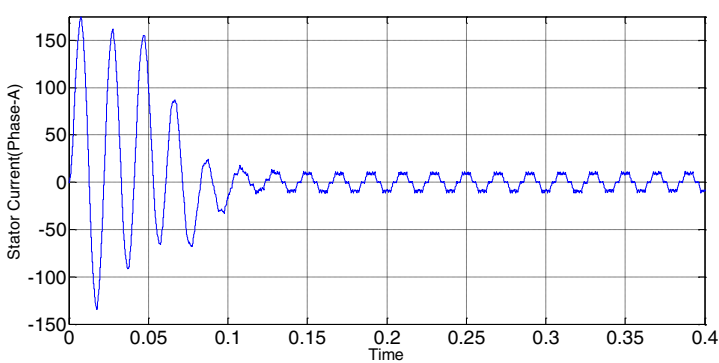

Fig.8. Stator current

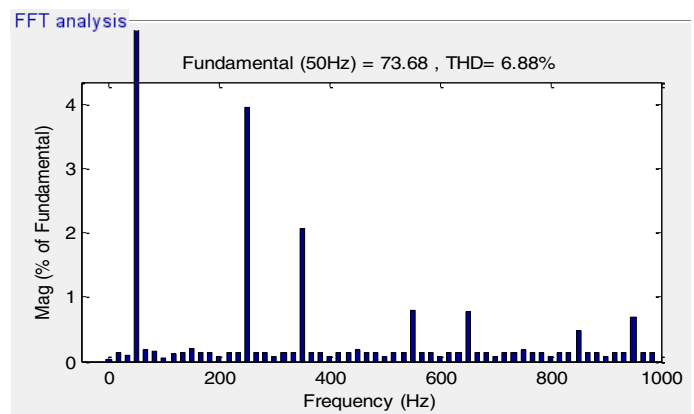

Fig.9. THD analysis of voltage 


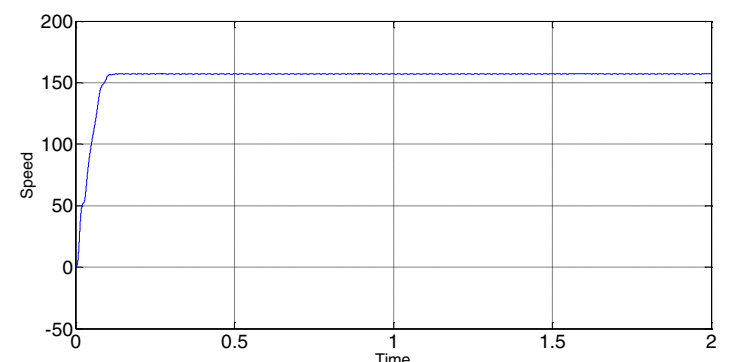

Fig.10. Speed of induction motor

\subsection{Three phase 7-Level semi cross switched multilevel inverter}

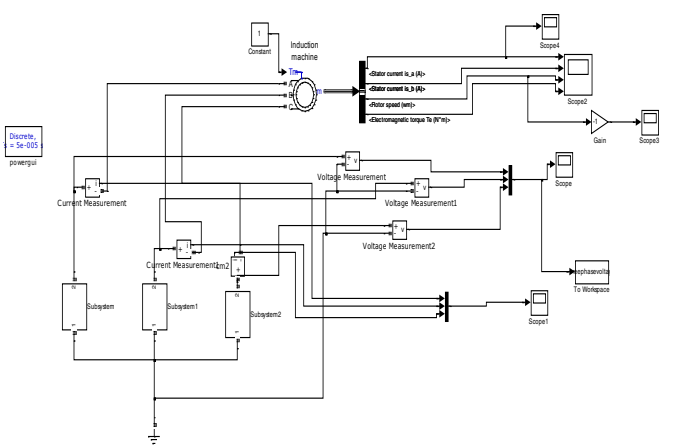

Fig.11. simulink diagram of three phase 7-level semi-cross switched multilevel inverter fed IM

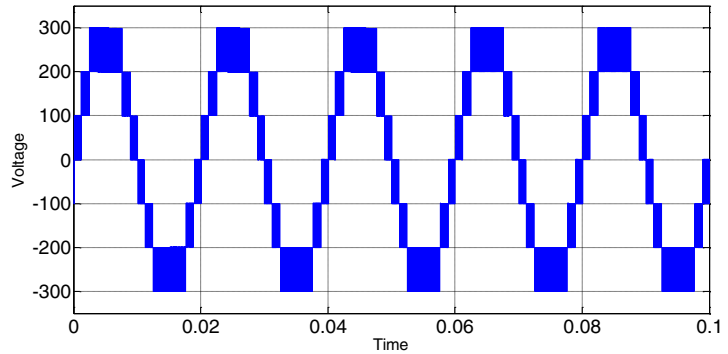

Fig.12. Phase voltage

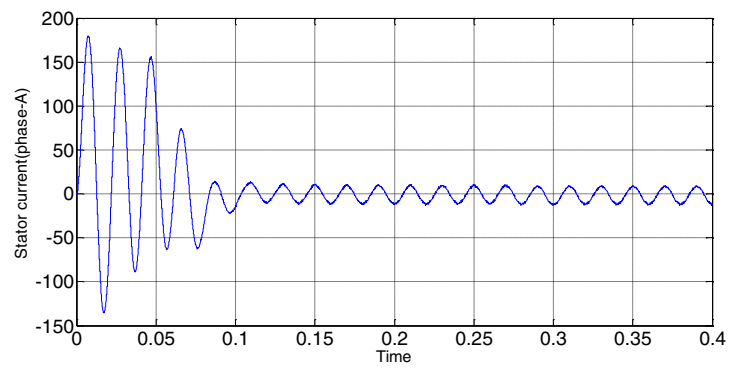

Fig.13. Stator current

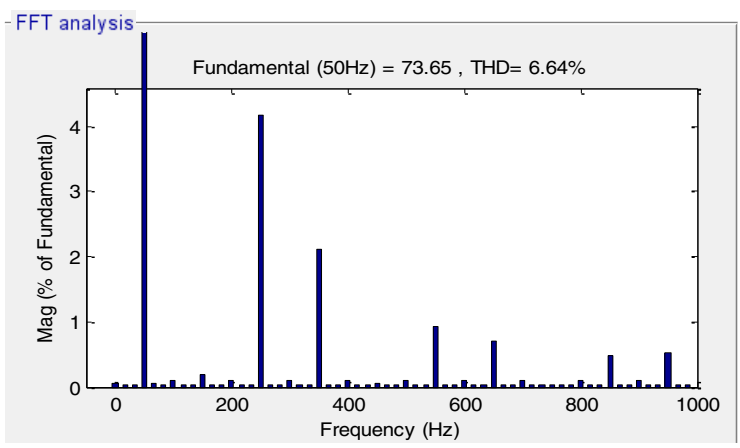

Fig.14. THD analysis of voltage

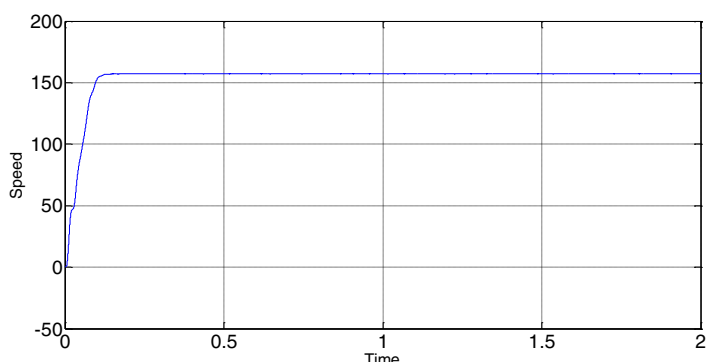

Fig.15. Speed of induction motor

Table 2. Comparison table for number of switching devices

\begin{tabular}{ccccc}
\hline \multirow{2}{*}{$\begin{array}{c}\text { OUTPUT } \\
\text { VOLTAG } \\
\text { E }\end{array}$} & \multicolumn{2}{c}{ NUMBER OF SWITCHING DEVICES } \\
\cline { 2 - 5 } LEVELS & \multicolumn{2}{c}{$\begin{array}{c}\text { Cascaded H-Bridge } \\
\text { MLI }\end{array}$} & \multicolumn{2}{c}{$\begin{array}{c}\text { semi-cross switched } \\
\text { MLI }\end{array}$} \\
\cline { 2 - 5 } & 1-Phase & 3-Phase & 1-Phase & 3-Phase \\
\hline 7 & 12 & 36 & 7 & 21 \\
LEVEL & & 36 & & 21 \\
\hline
\end{tabular}

Table 3. Comparison table for THD

\begin{tabular}{ccc}
\hline \multirow{2}{*}{$\begin{array}{c}\text { OUTPUT } \\
\text { VOLTAG } \\
\text { E } \\
\text { LEVELS }\end{array}$} & \multicolumn{2}{c}{ THD } \\
\cline { 2 - 3 } & $\begin{array}{c}\text { Cascaded H-Bridge } \\
\text { MLI }\end{array}$ & $\begin{array}{c}\text { semi-cross } \\
\text { switched MLI }\end{array}$ \\
\hline 7 & & \\
LEVEL & 6.68 & 6.64 \\
\hline
\end{tabular}

Table 4. Comparison table for losses

\begin{tabular}{ccc}
\multirow{2}{*}{$\begin{array}{c}\text { OUTPUT } \\
\text { VOLTAG } \\
\text { E }\end{array}$} & \multicolumn{2}{c}{ Losses/Phase(w) } \\
\cline { 2 - 3 } LEVELS & $\begin{array}{c}\text { Cascaded H-Bridge } \\
\text { MLI }\end{array}$ & $\begin{array}{c}\text { semi-cross switched } \\
\text { MLI }\end{array}$ \\
\cline { 2 - 3 } 7 & & \\
LEVEL & 7.34 & 4.28
\end{tabular}

\section{Conclusions}

The three phase 7- level cascaded H-bridge multilevel inverter and three phase 7-level semi cross switched multilevel inverter fed IM is simulated. From the analysis it clear that number of switches required, THD, and losses are also reduced for semicross switched multilevel inverter.

\section{Acknowledgement:}

Authors would like to thank All India Council for Technical Education (AICTE), Govt. of INDIA for sanction of grants under Reserach Promotion Scheme (RPS). Grant Number: 8-174/RIFD/RPS(Policy-1)/201819 dt: 22 Nov 2019.

\section{References:}

1. E.Avinash, L.Jebaraj “A New Variable Frequency Inverted Sine Carrier PWM Modulated Semi-Cross Switched Multilevel Inverter Topologies" 2016 
International Conference on Circuit, Power and Computing Technologies [ICCPCT]

2. M. Fracchia, T. Ghiara, M. Marchesoni, and M. Mazzucchelli, "Optimized modulation techniques or the gemeralized N-level converter," in proc. IEEE power electronics specialist Conf, 12051213, Madrid, Spain, 1992.

3. K. A. Corzine and J. R. Baker, "Reduced partscount multilevel retifiers," "IEEE Trans. Ind. Electron." vol.49, no.3, pp. 766-774, Aug 2002.

4. Srinivas Rao J., Srinivasa Varma, P., Suresh Kumar. T, International Journal of Power Electronics and Drive Systems, 9(3), pp. 12021213, 2018

5. F. Z. Peng, "A generalized multilevel inverter topology with self voltage balancing," "IEEE Trans. Ind. Applica," vol. 37, pp. 611-618, Mar./April 2004.

6. Suresh Kumar Tummala, Dhasharatha G, E3S Web of Conferences 87, 01030 (2019)

7. Tummala Suresh Kumar, Kosaraju Satyanarayana, Materials Today: Proceeding, 26 (2), 3228-3233, (2020).

8. Rajagiri A, MN Sandhya, Nawaz S, Suresh Kumar T, E3S Web of Conferences 8701004 (2019)

9. Suresh Kumar T, Sankar V, 2011 IEEE India Conference, INDICON, 2011

10. Bankupalli, P.T., Srikanth Babu, V., Suresh Kumar. $\mathrm{T}$, International Journal of Applied Engineering Research, 10(16), pp. 37057-37062, 2015.

11. Yogeswara Reddy B, Srinivas Rao J, Suresh Kumar T, Nagarjuna A, International Journal of Innovative Technology and Exploring Engineering, 8(11), 1194-1198, 2019.

12. Srinivasa Rao D, Sandhya Rani MN, Sarfaraz Naw az Syed and Suresh Kumar Tummala, E3S Web of Conferences 8701003 (2019) 